TRANSACTIONS OF THE

AMERICAN MATHEMATICAL SOCIETY

Volume 221, Number 1, 1976

\title{
DOCILITY AT INFINITY AND COMPACTIFICATIONS OF ANR'S
}

BY

\author{
R. B. SHER(1)
}

\begin{abstract}
Various conditions of contractibility and extensibility at $\infty$ for locally compact metric spaces are studied. These are shown to be equivalent if the space under consideration is an absolute neighborhood retract (ANR) and an ANR satisfying them is called docile at $\infty$. Docility at $\infty$ is invariant under proper homotopy domination. The ANR $X$ is docile at $\infty$ if and only if $F X$ (the Freudenthal compactification of $X$ ) is an ANR and $F X-X$ is unstable in $F X$; the inclusion of $X$ into $F X$ is a homotopy equivalence.
\end{abstract}

1. Introduction. In recent years there has been considerable interest in the geometry of noncompact spaces, particularly "at $\infty$ ". In this paper we consider various conditions of contractibility and extensibility at $\infty$ (alternatively, "at the ends") for locally compact metric spaces. These conditions are shown, in \$3, to be equivalent provided the space in question is an absolute neighborhood retract, and an absolute neighborhood retract satisfying them will be called docile at $\infty$. Docility at $\infty$ is shown to be invariant under proper homotopy domination.

In $\S 4$ it is shown that if $X$ is an absolute neighborhood retract which is docile at $\infty$, then $F X$, the Freudenthal compactification of $X$, is an absolute neighborhood retract, and that $E X$, the end-set of $X$, is unstable in $F X$. The converse is also shown to hold, and it is shown that the inclusion of $X$ into $F X$ is a homotopy equivalence.

2. Definitions, notations, and other preliminaries. Although not always required for our results, it will be convenient to adopt the convention for this paper that all spaces henceforth considered shall be locally compact separable and metrizable.

We use $I$ to denote the interval $[0,1]$ and $1_{X}$ to denote the identity function on the set $X$. The notations $\mathrm{Cl} A$, Int $A$, and $\mathrm{Fr} A$ are used for the closure, interior, and frontier of the set $A$ relative to some (understood from context) am-

Presented to the Society, March 20, 1975 under the title Docility at infinity and characterizations of APR's; received by the editors January 13, 1975 and, in revised form, June 5, 1975.

AMS (MOS) subject classifications (1970). Primary 54F40; Secondary 54C55, 54D35, 55C15, 55D99.

Key words and phrases. Absolute proper retract, docility at $\infty$, Freudenthal compactification, proper homotopy domination, unstable sets.

(1) This research was supported by a grant from the UNC-G Research Council. 
bient space. $X \in$ ANR (AR) means that $X$ is an absolute neighborhood retract (absolute retract) for metric spaces.

Map will always mean continuous function. The map $f: X \rightarrow Y$ is proper if $f^{-1}(C)$ is compact whenever $C$ is a compact subset of $Y$. If $Y_{0}, Y_{1}$, and $Y_{2}$ are subspaces of the space $Y$, then maps $f_{1}: X \rightarrow Y_{1}$ and $f_{2}: X \rightarrow Y_{2}$ are homotopic in $Y_{0}$ if there exists a map $F: X \times I \rightarrow Y_{0}$ such that $F(x, 0)=f_{1}(x)$ and $F(x, 1)=f_{2}(x)$ for all $x \in X$; similarly $f_{1}$ and $f_{2}$ are properly homotopic in $Y_{0}$ if the map $F$ can be chosen so as to be proper. If the maps $f, g: X \rightarrow Y$ are homotopic (properly homotopic) in $Y$, we say simply that $f$ and $g$ are homotopic (properly homotopic) and write $f \simeq g\left(f \simeq_{p} g\right)$. The map $f: X \rightarrow Y_{0} \subset Y$ is nullhomotopic in $Y$ if $f$ is homotopic in $Y$ to a constant map; $Y_{0}$ is contractible in $Y$ if the inclusion of $Y_{0}$ into $Y$ is nullhomotopic.

Let $\Sigma$ denote the class of separable locally compact metrizable spaces having compact quasi-component space. For example, if $X$ is a locally compact polyhedron, then $X \in \Sigma$ if and only if $X$ has only finitely many components. Then, if $X \in \Sigma$, the Freudenthal compactification [5] of $X$, denoted $F X$, is metrizable. We denote $F X-X$ by $E X$. If $X, Y \in \Sigma$, and $f: X \rightarrow Y$ is a proper map, then $f$ has a unique extension to a map $F f:(F X, E X) \rightarrow(F Y, E Y)$ [2, Lemma 4.2] or [8, Theorem 3]; such a map is end-preserving if $F f \mid E X$ is injective. An alternative treatment of $F X$ appears in [1].

3. Contractibility and extensibility at $\infty$. In this section we consider various contractibility and extensibility conditions at $\infty$ for the space $X$. These are shown to be equivalent if $X \in \mathrm{ANR}$, and they are also shown to be hereditary proper homotopy invariants in this case. These conditions describe a sort of maximal homotopy triviality at the ends of $X$.

(3.1) Definition. The space $X$ is contractible at $\infty(X$ is $C(E))$ if for each compact set $A \subset X$ there exists a compact set $B \subset X$ such that each component of $X-B$ is contractible in $X-A$.

Our next two results relate the above concept to the idea of the local contractibility of a set in relation to an ambient space. Definitions may be found in [4].

(3.2) THEOREM. If $X \in \Sigma$, then $X$ is $C(E)$ if $X$, regarded as a subspace of $F X$, is LC at e for every $e \in E X$. The converse holds provided $X$ is locally connected modulo a compact set.

Proof. Suppose first that $X$ is $L C$ at $e$ for all $e \in E X$. Let $A$ be a compact subset of $X$. Then, since $F X-A$ is an open subset of $F X$ containing $E X$, for each $e \in E X$ there exists an open set $V_{e}$ of $F X$ such that $e \in V_{e} \subset F X-A$, Fr $V_{e}$ is compact, and $X \cap V_{e}$ is contractible in $X \cap(F X-A)=X-A$. Let $B$ be a compact subset of $X$ such that each component of $X-B$ lies in one of the 
sets $V_{e}, e \in E X$. Then each component of $X-B$ is contractible in $X-A$, thereby showing that $X$ is $C(E)$.

Now suppose that $X$ is $C(E)$ and locally connected modulo a compact set. Let $e \in E X$ and let $U$ be a neighborhood of $e$ in $F X$. Then there exist pairwise disjoint open sets $U_{1}, U_{2}, \ldots, U_{n}$ in $F X$ such that $E X \subset \bigcup_{i=1}^{n} U_{i}$ and $e \in U_{1}$ $\subset U$. Then $A=F X-\bigcup_{i=1}^{n} U_{i}$ is a compact subset of $X$. Since $X$ is $C(E)$, there exists a compact set $B \subset X$ such that each component of $X-B$ is contractible in $X-A$. Since $X$ is locally connected modulo a compact set, there exists a connected open set $V$ of $F X$ such that $e \in V \subset F X-B$ and Fr $V \subset X$. Let $V^{\prime}$ $=V \cap X$. Then $V^{\prime}$ is a connected subset of $X-B$ and thus $V^{\prime}$ lies in some component of $X-B$. It follows that $V^{\prime}$ is contractible in $X-A=\bigcup_{i=1}^{n}\left(U_{i} \cap X\right)$. This implies that $V^{\prime}$ is contractible in $U_{1} \cap X \subset U \cap X$. This shows that $X$ is $L C$ at $e$, and the proof is complete.

The hypothesis that $X$ be locally connected modulo a compact set is necessary in the second part of the above proof. An example to show this is given by $X=\bigcup_{i=0}^{\infty} A_{i} \subset R^{2}$, where

$$
\begin{aligned}
& A_{0}=\{(x, 0) \mid 0 \leqslant x \leqslant 1 / 2\}, \quad A_{1}=\{(0, y) \mid y \geqslant 0\}, \text { and } \\
& A_{i}=\{(1 / i, y) \mid y \geqslant 0\} \quad \text { for } i=2,3, \ldots
\end{aligned}
$$

Now, if $A$ is a subset of the metric space $X$ and $\mathrm{Cl} A$ is compact, then $A$ is ULC if and only if $A$ is $L C$ rel $X[4$, p. 615]. Thus Theorem 3.2 immediately yields the following. In its statement, a metric on $X$ compatible with $F X$ is the restriction to $X$ of a metric on $F X$.

(3.3) CoRollary. Let $X \in \Sigma$ be equipped with a metric compatible with $F X$. Then $X$ is $C(E)$ and $L C$ if and only if $X$ is ULC.

The following shows that the property of a space being $C(E)$ is invariant under proper homotopy domination.

(3.4) THEorem. If $X \leqslant_{p} Y$ and $Y$ is $C(E)$, then $X$ is $C(E)$.

Proof. Suppose $Y$ is $C(E)$ and $X \leqslant_{p} Y$. Let $h: X \rightarrow Y, g: Y \rightarrow X$, and $F: X \times I \rightarrow X$ be proper maps such that $F(x, 0)=x$ and $F(x, 1)=g h(x)$ for all $x \in X$. If $A$ is a compact subset of $X$ then, letting $A^{\prime}=g^{-1}(A)$, there exists a compact set $B^{\prime} \subset Y$ such that each component of $Y-B^{\prime}$ is contractible in $Y-$ $A^{\prime}$. Let $B$ be a compact subset of $X$ such that $F^{-1}(A) \subset B \times I$ and $h^{-1}\left(B^{\prime}\right) \subset$ $B$. In order to show that $X$ is $C(E)$, it will suffice to show that each component of $X-B$ is contractible in $X-A$.

Let $P$ be a component of $X-B$. Then $h(P)$ lies in a component $P^{\prime}$ of $Y-B^{\prime}$. By our choice of $B^{\prime}$, there exists a point $p_{0}^{\prime} \in Y$ and a map $G: P^{\prime} \times I$ 
$\rightarrow Y$ such that $G\left(P^{\prime} \times I\right) \subset Y-A^{\prime}, G\left(p^{\prime}, 0\right)=p^{\prime}$, and $G\left(p^{\prime}, 1\right)=p_{0}^{\prime}$ for all $p^{\prime} \in P^{\prime}$. Define $H: P \times I \longrightarrow X$ by

$$
H(p, t)= \begin{cases}F(p, 2 t) & \text { if } 0 \leqslant t \leqslant 1 / 2, \\ g(G(h(p), 2 t-1)) & \text { if } 1 / 2 \leqslant t \leqslant 1,\end{cases}
$$

for all $p \in P$ and $t \in I$. Then $H$ is easily seen to be a contraction of $P$ in $X-A$, thereby completing the proof.

(3.5) Definition. The space $X$ is $C$-connected at $\infty(X$ is $C-C(E))$ if for each compact set $A \subset X$ there exists a compact set $B \subset X$ such that if $C_{0}$ is a compactum and $f: C_{0} \rightarrow X-B$ is a map carrying $C_{0}$ into a component of $X-$ $B$, then $f$ is nullhomotopic in $X-A$.

(3.6) Definition. The space $X$ is $K$-connected at $\infty(X$ is $K-C(E))$ if for each compact set $A \subset X$ there exists a compact set $B \subset X$ such that if $K_{0}$ is a compact polyhedron and $f: K_{0} \rightarrow X-B$ is a map carrying $K_{0}$ into a component of $X-B$, then $f$ is nullhomotopic in $X-A$.

Obviously $X$ is $K-C(E)$ if it is $C-C(E)$. That the two notions differ in general can be seen from the following example. Let $W$ be a "Warsaw circle" and let $w_{0}$ be a point of $W$ at which $W$ is locally connected. Let $X$ be the space obtained by attaching to the nonnegative reals a homeomorphic copy $W_{i}$ of $W$ at each nonnegative integer $i$, identifying the integer $i$ with the point in $W_{i}$ corressponding to $w_{0}$. Since $w_{i}$ is not contractible in $X$ for $i=0,1, \ldots$, it follows that $X$ is not $C-C(E)$. However, using the fact that the continuous image of a compact polyhedron is locally connected, it can easily be verified that $X$ is $K$ $C(E)$. By the following, such disparity cannot occur if the space under consideration is an absolute neighborhood retract.

(3.7) THEOREM. If $X \in A N R$, then $X$ is $C-C(E)$ if and only if $X$ is $K-$ $C(E)$.

Proof. It is necessary to show that if $X \in \mathrm{ANR}$ and $X$ is $K-C(E)$, then $X$ is $C-C(E)$. To this end, let $A$ be a compact subset of $X$, and let $B$ be a compact subset of $X$ such that any map of a compact polyhedron into a component of $X-B$ is nullhomotopic in $X-A$. Let $X_{0}$ be a component of $X-B$. Then $X_{0}$ is open in $X$. Hence $X_{0} \in \mathrm{ANR}$. Now, let $C_{0}$ be a compactum and $f: C_{0} \rightarrow$ $X_{0}$ a map. We shall show that $f$ is nullhomotopic in $X-A$, thereby showing that $X$ is $C-C(E)$.

Since $X_{0} \in$ ANR, there exists a "bridge" for $f[6$, Chapter IV, §8]. More precisely, there exists an open cover $\alpha$ of $C_{0}$ such that if $\beta$ is any locally finite open refinement of $\alpha$, if $N_{\beta}$ is the nerve of $\beta$, and if $\phi_{\beta}: C_{0} \rightarrow N_{\beta}$ is a canonical map, then there exists a map $g_{\beta}: N_{\beta} \rightarrow X_{0}$ such that $f \simeq g_{\beta} \phi_{\beta}$. Since $C_{0}$ is com- 
pact, we may choose such a $\beta$ which is a finite refinement of $\alpha$, so that $N_{\beta}$ is a compact polyhedron. Then, by our choice of $B, g_{\beta}$ is nullhomotopic in $X-A$. This implies that $f$ is nullhomotopic in $X-A$, thereby completing the proof.

(3.8) Definition. The space $X$ is an absolute extensor at $\infty$ for compacta $(X \in A E C(E))$ if for each compact set $A \subset X$ there exists a compact set $B \subset X$ such that if $D_{0} \subset C_{0}$ are compacta and $f: D_{0} \rightarrow X-B$ is a map carrying $D_{0}$ into a component of $X-B$, then there exists a map $f^{*}: C_{0} \rightarrow X-A$ such that $f^{*}(d)=f(d)$ for all $d \in D_{0}$.

Clearly $X$ is $C-C(E)$ if $X \in A E C(E)$. But the converse does not generally hold, as seen from the following example. Let $F$ denote the cone on the set $\{0,1 / 2,1 / 3,1 / 4, \ldots\} \subset I$ (a fan) with vertex $v$. Let $X$ be the space obtained by attaching to $R^{+}$, the nonnegative reals, a homeomorphic copy $F_{i}$ of $F$ at each nonnegative integer $i$, identifying the integer $i$ with the point $v_{i} \in F_{i}$ corresponding to $v$. It is easy to see that $R^{+}$is a strong deformation retract of $X$ (under a homotopy which contracts each $F_{i}$ to $\left.\left\{v_{i}\right\}\right)$, and that this implies that $X$ is $C-$ $C(E)$. However, $X \notin A E C(E)$, for if $D_{0}=\{0,1 / 2,1 / 3,1 / 4, \ldots\} \subset I=C_{0}$ and $i$ is a nonnegative integer, then there is a map of $D_{0}$ into $F_{i}$ (corresponding to the inclusion of $D_{0}$ into $F$ ) which cannot be extended to a map of $C_{0}$ into $X$. The following result shows that this sort of behavior cannot occur if $X \in$ ANR.

(3.9) THEOREM. If $X \in A N R$, then $X$ is $C-C(E)$ if and only if $X \in A E C(E)$.

Proof. It is necessary to show that if $X \in \mathrm{ANR}$ and $X$ is $C-C(E)$, then $X \in A E C(E)$. For this purpose, let $A$ be a compact subset of $X$, and let $B$ be a compact subset of $X$ such that if $C^{\prime}$ is a compactum and $f^{\prime}: C^{\prime} \rightarrow X-B$ is a map carrying $C^{\prime}$ into a component of $X-B$, then $f^{\prime}$ is nullhomotopic in $X-A$. Let $X_{0}$ be a component of $X-B$, let $D_{0} \subset C_{0}$ be compacta, and let $f: D_{0} \rightarrow$ $X_{0}$ be a map. To show that $X \in A E C(E)$, it will suffice to show that there exists a map $f^{*}: C_{0} \rightarrow X-A$ such that $f^{*}(d)=f(d)$ for all $d \in D_{0}$.

By our choice of $B$, there exists a map $F: D_{0} \times I \rightarrow X-A$ and a point $x_{0} \in X-A$ such that $F(d, 0)=x_{0}$ and $F(d, 1)=f(d)$ for all $d \in D_{0}$. Since $A$ is compact, $X-A$ is an open subset of $X$, and hence $(X-A) \in$ ANR. By the Borsuk Homotopy Extension Theorem [3, Chapter IV, §8] there exists a map $G$ : $C_{0} \times I \rightarrow X-A$ such that $G(c, 0)=x_{0}$ for all $c \in C_{0}$, and $G(d, t)=F(d, t)$ for all $d \in D_{0}, t \in I$. The proof is now completed by defining $f^{*}: C_{0} \rightarrow X-A$ by $f^{*}(c)=G(c, 1)$ for all $c \in C_{0}$.

(3.10) Definition. The proper map $f: X \rightarrow Y$ properly factors through the space $Z$ if there exist proper maps $g: X \rightarrow Z$ and $h: Z \rightarrow Y$ such that $f=$ hg. If $f: X \rightarrow Y \subset Y^{\prime}$ is a proper map, we say that $f$ is properly nullhomotopic in $Y^{\prime}$ if there exists a map $f^{\prime}: X \rightarrow Y^{\prime}$ such that $f \simeq_{p} f^{\prime}$ in $Y^{\prime}$ and $f^{\prime}$ properly factors through a tree. (By tree we mean locally finite connected and simply connected 1-complex.) Suppose $X \subset X^{\prime}$ are spaces. Then $X$ is said to be properly 
contractible in $X^{\prime}$ if the inclusion of $X$ into $X^{\prime}$ is properly nullhomotopic in $X^{\prime}$.

(3.11) Definition. The space $X$ is properly contractible at $\infty$ ( $X$ is $P C(E)$ ) if for each compact set $A \subset X$ there exists a compact set $B \subset X$ such that the closure of each component of $X-B$ is properly contractible in $X-A$.

Our immediate goal is to prove that $X$ is $P C(E)$ if $X \in A E C(E)$. But first we shall require two lemmas.

(3.12) Lemma. If $X, Y \in \Sigma, g: X \rightarrow Y$ is a proper end-preserving map, and $B$ is a compact subset of $X$ such that $X$ is locally connected modulo $B$, then there exists a compact subset $D$ of $Y$ such that if $Y_{0}$ is a component of $Y-D$, then $g^{-1}\left(Y_{0}\right)$ lies in a component of $X-B$.

Proof. Suppose that the hypothesis of the Lemma holds, but that the conclusion fails to hold. Since $X-B$ is locally connected and $X \in \Sigma, X-B$ has only finitely many unbounded components and the union of $B$ and the bounded components of $X-B$ is compact. (As used here, bounded means having compact closure.) By this latter fact, there exists a compact subset $D_{1}$ of $Y$ such that $g^{-1}\left(D_{1}\right)$ contains the union of $B$ and the bounded components of $X-B$. Let $D_{1} \subset D_{2} \subset \cdots$ be compact subsets of $Y$ such that $Y=\bigcup_{i=1}^{\infty} D_{i}$.

For $i=1,2, \ldots$ there exists a component $Y_{0}^{i}$ of $Y-D_{i}$ such that $g^{-1}\left(Y_{0}^{i}\right)$ intersects at least two components of $X-B$, and these components of $X-B$ must be unbounded. We may assume, by selecting a subsequence of $D_{1}, D_{2}, \ldots$ if necessary and relabeling, that there exist components $U_{1}$ and $U_{2}$ of $X-B$ such that $U_{1} \cap g^{-1}\left(Y_{0}^{i}\right) \neq \varnothing \neq U_{2} \cap g^{-1}\left(Y_{0}^{i}\right)$ for $i=1,2, \ldots$ If $i=1,2$, ..., let $p_{i} \in U_{1} \cap g^{-1}\left(Y_{0}^{i}\right)$ and $q_{i} \in U_{2} \cap g^{-1}\left(Y_{0}^{i}\right)$. Again choosing subsequences and relabeling if necessary, we may assume that $\left\{p_{i}\right\}_{i=1}^{\infty} \rightarrow e \in E X$ and $\left\{q_{i}\right\}_{i=1}^{\infty} \rightarrow e^{\prime} \in E X$. Since $U_{1}$ and $U_{2}$ are disjoint open subsets of $X$ having compact frontier, $e \neq e^{\prime}$. But $g\left(p_{i}\right)$ and $g\left(q_{i}\right)$ lie in the same component of $Y-$ $D_{i}$, namely $Y_{0}^{i}$, and hence the sequences $\left\{g\left(p_{i}\right)\right\}_{i=1}^{\infty}$ and $\left\{g\left(q_{i}\right)\right\}_{i=1}^{\infty}$ have a com. mon limit in $E Y$. This contradicts the hypothesis that $g$ is end-preserving, completing the proof.

The hypothesis that $X$ be locally connected modulo $B$ is necessary in Lemma 3.12. To see this, let $X=\bigcup_{i=0}^{\infty} X_{i} \subset R^{2}$, where $X_{0}=\{(x, y) \mid 0 \leqslant x \leqslant 1 / 2$, $y=0\}, X_{1}=\{(x, y) \mid x=0, y \geqslant 0\}$, and $X_{n}=\{(x, y) \mid x=1 / n, 0 \leqslant y \leqslant n\}$ for $n=2,3, \ldots$. Let $Y=X_{1}$ and define $g: X \rightarrow Y$ by $g(x, y)=(x, 0)$ for all $x \in X$. Then $g$ is an end-preserving proper map, but if $B=X_{0}, D$ is any compact subset of $Y$, and $Y_{0}$ is the unbounded component of $Y-D$, then $g^{-1}\left(Y_{0}\right)$ will intersect infinitely many components of $X-B$. Accordingly, to apply Lemma 3.12 to the proof of Theorem 3.14 , we shall require the following lemma. Its proof is routine, and shall be omitted.

(3.13) Lemma. Suppose B is a closed subset of the space $X$ such that for 
any closed set $C \subset I$ and map $f: C \rightarrow X-B$ carrying $C$ into a component of $X-B$, there exists a map $f^{*}: I \rightarrow X$ such that $f^{*}(c)=f(c)$ for all $c \in C$. Then each component of $X-B$ is locally connected.

Recall that if $f: X \rightarrow Y$ is a map, then the mapping cylinder $\left(M_{f}\right)$ of $f$ is obtained from the disjoint union of $X \times I$ and $Y((X \times I) \oplus Y)$ by identifying $(x, 1) \in X \times I$ with $f(x) \in Y$. Let $\Pi:(X \times I) \oplus Y \rightarrow M_{f}$ denote the natural projection. If $f$ is proper, then so is $\Pi$, and $M_{f}$ is locally compact, separable, and metrizable. We follow the usual convention of regarding $X$ and $Y$ as subspaces of $M_{f}$, identifying $X$ with $\Pi(X \times\{0\})$ and $Y$ with $\Pi(Y)$.

We are now prepared to proceed to Theorem 3.14 .

(3.14) THEOREM. If $X \in A E C(E)$, then $X$ is $P C(E)$.

Proof. Suppose $X \in A E C(E)$, and let $A$ be a compact subset of $X$. Letting $A=B_{0}$, inductively choose compact sets $B_{0} \subset B_{1} \subset \cdots \subset X$ such that $X$ $=\bigcup_{i=1}^{\infty} B_{i}$ and such that if $i=1,2, \ldots, D_{0} \subset C_{0}$ are compacta, and $f: D_{0}$ $\rightarrow X-B_{i}$ is a map carrying $D_{0}$ into a component of $X-B_{i}$, then there exists a map $f^{*}: C_{0} \rightarrow X-B_{i-1}$ such that $f^{*}(d)=f(d)$ for all $d \in D_{0}$. We may further assume that $B_{2} \subset$ Int $B_{3}$, so that there exists a compact subset $B$ of $X$ such that $B_{2} \subset$ Int $B \subset B \subset$ Int $B_{3}$. Let $Y$ be the closure of one of the components of $X-B$. In order to show that $X$ is $P C(E)$, it will suffice to show that $Y$ is properly contractible in $X-A$. Since this is immediate if $Y$ is compact, we assume henceforth that $Y$ is noncompact.

By [9, Theorem 2.3] there exists a tree $T$ and a proper map $g: Y \rightarrow T$ such that $E T \cong E Y$ and $F g \mid E Y$ is an embedding. Let $M_{g}$ denote the mapping cylinder of $g$ and $\Pi:(Y \times I) \oplus T \rightarrow M_{g}$ the canonical projection. To show that $Y$ is properly contractible in $X-A$, it clearly suffices to find a proper map $\Phi$ : $M_{g} \rightarrow X-A$ such that $\Phi(y)=y$ for all $y \in Y$.

Note that, by Lemma 3.13, if $\hat{Y}$ is the component of $X-B_{2}$ containing $Y$, then $\hat{Y}$ is locally connected. Furthermore, since $B \subset$ Int $B_{3} \subset B_{3} \subset B_{4} \subset \cdots$, $Y-B_{j}$ is an open subset of $\hat{Y}$ for $j=3,4, \ldots$, and thus $Y-B_{j}$ is locally connected. Also, $B_{j} \cap Y$ is a compact subset of $Y$ since $Y$ is closed. Hence, we may apply Lemma 3.12 to obtain compact sets $\varnothing=T_{0} \subset T_{1} \subset \cdots \subset T$ such that $T=\bigcup_{i=0}^{\infty} T_{i}$ and such that if $Z$ is the closure of one of the components of $T$ $T_{i}$, for $i=0,1, \ldots$, then $g^{-1}(Z)$ lies in a component of $Y-B_{i+2}$. We may further assume, by "expanding" the $T_{i}$ 's if necessary, that the sets $T_{1}, T_{2}, \ldots$ are connected, that $T_{i} \subset$ Int $T_{i+1}$, that $T_{i+1}-T_{i}$ has only finitely many components, and that the components of $T_{i+1}-T_{i}$ have pairwise disjoint closures in $T$, for $i=0,1, \ldots$ If $i=1,2, \ldots$, let $K^{i}$ denote the closure of $T_{i}-T_{i-1}$ and let $K_{1}^{i}, K_{2}^{i}, \ldots, K_{n_{i}}^{i}$ denote the components of $K^{i}$; let $L^{i}=g^{-1}\left(K^{t}\right)$, and $L_{j}^{i}=g^{-1}\left(K_{j}^{i}\right)$, where $j=1,2, \ldots, n_{i}$. Then $L_{1}^{i}, L_{2}^{i}, \ldots, L_{n_{i}}^{i}$ are pairwise dis- 
joint compacta and each $L_{j}^{i}$ lies in a component of $X-B_{i+1}$. For $i=1,2$, $\ldots$, let $P^{i}=\Pi\left(L^{i} \times I\right) \subset M_{g}$ and let $P_{j}^{i}=\Pi\left(L_{j}^{i} \times I\right) \subset M_{g}$ for $j=1,2$, $\ldots, n_{i}$.

Now $P_{j}^{i} \cap Y=L_{j}^{i}$ lies in a component of $X-B_{i+1}$, for $i=1,2, \ldots$ Hence, if $i=1,3,5, \ldots$, and $j=1,2, \ldots, n_{i}$, there exists a map $\Phi_{j}^{i}: P_{j}^{i} \rightarrow$ $X-B_{i}$ such that $\Phi_{j}^{i}(y)=y$ for all $y \in L_{j}^{i}$. Since the sets $P_{1}^{i}, P_{2}^{i}, \ldots, P_{n_{i}}^{i}$ are pairwise disjoint and closed, we may glue the maps $\Phi_{1}^{i}, \Phi_{2}^{i}, \ldots, \Phi_{n_{i}}^{i}$ to obtain a map $\Phi^{i}: P^{i} \rightarrow X-B_{i}$ such that $\Phi^{i}(y)=y$ for all $y \in L^{i}$.

Now, if $i=2,4,6, \ldots$, and $j=1,2, \ldots, n_{i}$, let

$$
\hat{P}_{j}^{i}=\left(P_{j}^{i} \cap P^{i-1}\right) \cup L_{j}^{i} \cup\left(P_{j}^{i} \cap P^{i+1}\right) \subset P_{j}^{i},
$$

and define $\Psi_{j}^{i}: \hat{P}_{j}^{i} \rightarrow X-B_{i-1}$ by

$$
\Psi_{j}^{i}(p)= \begin{cases}\Phi^{i-1}(p) & \text { if } p \in P_{j}^{i} \cap P^{i-1} \\ p & \text { if } p \in L_{j}^{i} \\ \Phi^{i+1}(p) & \text { if } p \in P_{j}^{i} \cap P^{i+1}\end{cases}
$$

for all $p \in \hat{P}_{j}^{i}$. Then $\Psi_{j}^{i}$ is continuous and carries $\hat{P}_{j}^{i}$ into a single component of $X-B_{i-1}$. Hence there exists a map $\Phi_{j}^{i}: P_{j}^{i} \rightarrow X-B_{i-2}$ such that $\Phi_{j}^{i}(p)=$ $\Psi_{j}^{i}(p)$ for all $p \in \hat{P}_{j}^{i}$. We now glue the maps $\Phi_{1}^{i}, \Phi_{2}^{i}, \ldots, \Phi_{n_{i}}^{i}$ together to obtain a map $\Phi^{i}: P^{i} \rightarrow X-B_{i-2}$.

Define $\Phi: M_{g} \rightarrow X-A$ by $\Phi(p)=\Phi^{i}(p)$ if $p \in P^{i}$. Then $\Phi$ is proper and $\Phi(y)=y$ for all $y \in Y$, so $\Phi$ is the map required for the completion of the proof.

If $X \subset X^{\prime}$ and $X$ is properly contractible in $X^{\prime}$, then $X$ is contractible in $X^{\prime}$. Thus, we have the following

(3.15) THEOREM. If $X$ is $P C(E)$, then $X$ is $C(E)$.

Now, combining previous results, we obtain the following equivalence.

(3.16) THEOREM. If $X \in A N R$, then the following are equivalent.

(i) $X$ is $C(E)$.

(ii) $X$ is $K-C(E)$.

(iii) $X$ is $C-C(E)$.

(iv) $X \in A E C(E)$.

(v) $X$ is $P C(E)$.

(3.17) Definition. If $X \in A N R$ and $X$ satisfies (i)-(v) of Theorem 3.16, then $X$ is said to be docile at $\infty$. 
The following is immediate from Theorem 3.4.

(3.18) THEOREM. If $X, Y \in A N R$ and $X \leqslant_{p} Y$, then $X$ is docile at $\infty$ if $Y$ is docile at $\infty$.

In the following section we shall examine the notion of docility as it applies to the Freudenthal compactification of an ANR of the class $\Sigma$.

4. The Freudenthal compactification of docile ANR's. In general, it is not the case that $F X \in$ ANR if $X \in$ ANR and $X \in \Sigma$. In this section we shall show that this conclusion can be reached if $X$ is docile at $\infty$, and we shall show that the converse holds if $E X$ is an unstable subset of $F X$.

(4.1) Lemma. Suppose that $X \in A N R, X \in \Sigma$, and $X$ is docile at $\infty$. Then if $K \subset L$ are compacta and $g: K \rightarrow F X$ is a map, $g$ can be extended over a neighborhood $U$ of $K$ in $L$ to a map $h: U \rightarrow F X$ such that $h^{-1}(E X)=g^{-1}(E X)$.

Proof. The idea of the proof is very much the same as that of the proof of Theorem 3.14. Because of this similarity, we take the liberty of avoiding ballooning of notation by assuming that $X$ has precisely one end. The modifications necessary for the general proof will be clear.

Let $B_{0} \subset B_{1} \subset \cdots$ be compact subsets of $X$ such that $X=\bigcup_{i=0}^{\infty} B_{i}$, $X-B_{i}$ is connected for $i=0,1, \ldots$, and such that if $D_{0} \subset C_{0}$ are compacta, $j=1,2, \ldots$, and $f: D_{0} \rightarrow X-B_{j}$ is a map, then there exists a map $f^{*}: C_{0}$ $\rightarrow X-B_{j-1}$ such that $f^{*}(d)=f(d)$ for all $d \in D_{0}$. Let $K_{0}=g^{-1}(E X)$, and let $N_{1}, N_{2}, \ldots$ be compact neighborhoods of $K_{0}$ in $L$ such that $K_{0}=\bigcap_{i=1}^{\infty} N_{i}$, $N_{i+1} \subset$ Int $N_{i}$, and $g\left(K \cap N_{i}\right) \subset F X-B_{i+1}$ for $i=1,2, \ldots$ Let $M_{i}=$ $\mathrm{Cl}\left(N_{i}-N_{i+1}\right)$ for $i=1,2, \ldots$, and let if $n \in K_{0}$,

For every odd positive integer $i$, let $\Phi_{i}: M_{i} \rightarrow X-B_{i}$ be a map such that $\Phi_{i}(m)=g(m)$ for all $m \in M_{i}^{\prime}$. Now, fixing $i$ to be an even positive integer, let $M_{i}^{\prime \prime}=\left(M_{i} \cap M_{i-1}\right) \cup M_{i}^{\prime} \cup\left(M_{i} \cap M_{i+1}\right)$, and define $\Psi_{i}: M_{i}^{\prime \prime} \rightarrow X-B_{i-1}$ by

$$
\Psi_{i}(m)= \begin{cases}\Phi_{i-1}(m) & \text { if } m \in M_{i} \cap M_{i-1}, \\ g(m) & \text { if } m \in M_{i}^{\prime}, \\ \Phi_{i+1}(m) & \text { if } m \in M_{i} \cap M_{i+1},\end{cases}
$$

for all $m \in M_{i}^{\prime \prime}$. There exists a map $\Phi_{i}: M_{i} \rightarrow X-B_{i-2}$ such that $\Phi_{i}(m)=$ $\Psi_{i}(m)$ for all $m \in M_{i}^{\prime \prime}$. Now, define the map $h^{\prime}: N_{1} \rightarrow F X$ by

$$
h^{\prime}(n)= \begin{cases}\Phi_{i}(n) & \text { if } n \in M_{i}, \\ g(n) & \text { if } n \in K_{0},\end{cases}
$$

for all $n \in N_{1}$. Let $Z=L-$ Int $N_{1}$, let $Z_{0}=(K \cap Z) \cup \operatorname{Fr} N_{1}$, and define $h^{\prime \prime}$ : $Z_{0} \rightarrow X$ by 


$$
h^{\prime \prime}(z)= \begin{cases}g(z) & \text { if } z \in K \cap Z, \\ h^{\prime}(z) & \text { if } z \in \operatorname{Fr} N_{1},\end{cases}
$$

for all $z \in Z_{0}$. Then, since $Z_{0}$ is closed in $Z$ and $X \in A N R$, there exist a neighborhood $U_{0}$ of $Z_{0}$ in $Z$ and a map $h^{*}: U_{0} \rightarrow X$ such that $h^{*}(z)=h^{\prime \prime}(z)$ for all $z \in Z_{0}$. Let $U=U_{0} \cup N_{1}$. Then $U$ is a neighborhood of $K$ in $L$, and the required map $h: U \rightarrow F X$ may be defined by

$$
h(u)= \begin{cases}h^{*}(u) & \text { if } u \in U_{0}, \\ h^{\prime}(u) & \text { if } u \in N_{1},\end{cases}
$$

for all $u \in U$.

If $Z$ is a space and $Z^{\prime} \subset Z$, then $Z^{\prime}$ is an unstable subset of $Z$ if there exists a homotopy $H: Z \times I \rightarrow Z$ such that $H(z, 0)=z$ and $H(z, t) \notin Z^{\prime}$ for all $z \in Z$ and $0<t \leqslant 1$.

The following generalizes a result of Kozlowski [7].

(4.2) THEOREM. If $X \in A N R$ and $X \in \Sigma$, then $X$ is docile at $\infty$ if and only if $F X \in A N R$ and $E X$ is an unstable subset of $F X$.

Proof. Suppose first that $X \in \mathrm{ANR}, X \in \Sigma$, and $X$ is docile at $\infty$. By Lemma 4.1, $F X$ is an absolute neighborhood extensor for compact metric spaces, and hence $F X \in A N R$. It remains to be shown that $E X$ is an unstable subset of $F X$. Let $K=F X \times\{0\} \subset F X \times I=L$, and define $g: K \rightarrow F X$ by $g(x, 0)=x$ for all $x \in F X$. By Lemma 4.1, there exists a neighborhood $U$ of $K$ in $L$ and a map $h: U \rightarrow F X$ such that $h(x, 0)=x$ for all $x \in F X$ and $h^{-1}(E X)=g^{-1}(E X)$ $=E X \times\{0\}$. Choose $t_{0} \in(0,1]$ such that $F X \times\left[0, t_{0}\right] \subset U$, and define $\Phi:$ $F X \times I \rightarrow F X$ by $\Phi(x, t)=h\left(x, t t_{0}\right)$ for all $(x, t) \in F X \times I$. Then $\Phi(x, 0)=$ $x$ for all $x \in F X$ and, if $t>0, \Phi(x, t) \in F X-E X$. This shows that $E X$ is an unstable subset of $F X$.

For the converse, suppose now that $F X \in \mathrm{ANR}$ and that $E X$ is an unstable subset of $F X$. Let $\Phi: F X \times I \rightarrow F X$ be a homotopy such that $\Phi(x, 0)=x$ for all $x \in F X$ and $\Phi(x, t) \notin E X$ for all $x \in F X$ and $t \in(0,1]$. To show that $X$ is docile at $\infty$, it suffices to show that $X$ is $C-C(E)$. To this end, let $A$ be a com. pact subset of $X$, and let $A^{\prime}$ be a compact subset of $X$ such that $A \subset \operatorname{Int} A^{\prime}$.

Since $F X \in A N R, F X$ is $L C$ at $e$ for all $e \in E X$. It follows that there exist open sets $U_{1}, U_{2}, \ldots, U_{n}$ in $F X$ covering $E X$ such that $U_{t}$ is contractible in $F X$ $A^{\prime}$ for $i=1,2, \ldots, n$. Let $B$ be a compact subset of $X$ such that each component of $X-B$ lies in one of the sets $U_{1}, U_{2}, \ldots, U_{n}$. We will show that any map of a compactum into a component of $X-B$ is nullhomotopic in $X-A$, thereby completing the proof.

Let $C_{0}$ be a compactum and $f: C_{0} \rightarrow X-B$ a map carrying $C_{0}$ into a 
component of $X-B$. Then $f\left(C_{0}\right) \subset U_{j}$ for some $j$. Hence there exists a map $F: C_{0} \times I \rightarrow F X-A^{\prime}$ and a point $p_{0} \in F X$ such that $F(c, 0)=f(c)$ and $F(c, 1)=p_{0}$ for all $c \in C_{0}$. Since $A \cap \operatorname{Cl}\left(F X-A^{\prime}\right)=\varnothing$, we may choose $t_{0} \in$ $(0,1]$ such that $\Phi\left(\mathrm{Cl}\left(F X-A^{\prime}\right) \times\left[0, t_{0}\right]\right) \subset F X-A$. Now define $G: C_{0} \times I$ $\rightarrow X-A$ by $G(c, t)=\Phi\left(F(c, t), t t_{0}\right)$ for all $(c, t) \in C_{0} \times I$. Then $G$ is a homotopy joining $f$ with a constant map in $X-A$, and the proof is complete.

(4.3) Lемма. If $Z^{\prime}$ is an unstable subset of the space $Z$, then the inclusion of $Z-Z^{\prime}$ into $Z$ is a homotopy equivalence.

Proof. Let $H: Z \times I \rightarrow Z$ be a map such that $H(z, 0)=z$ and $H(z, t) \notin$ $Z^{\prime}$ for all $z \in Z$ and $0<t \leqslant 1$. Let $i: Z-Z^{\prime} \rightarrow Z$ be the inclusion and define $f: Z \rightarrow Z-Z^{\prime}$ by $f(z)=H(z, 1)$ for all $z \in Z$. Then, defining $F_{1}:\left(Z-Z^{\prime}\right) \times$ $I \rightarrow Z-Z^{\prime}$ by $F_{1}(z, t)=H(z, t)$ for all $z \in Z-Z^{\prime}$ and $t \in I$, we see that $F_{1}$ is a homotopy joining $1_{z-z}$ and $f i$. Since $H$ is a homotopy joining $1_{z}$ and if, the proof is complete.

The following is immediate from Lemma 4.3 and Theorem 4.2.

(4.4) Theorem. If $X \in A N R, X \in \Sigma$, and $X$ is docile at $\infty$, then the inclusion of $X$ into $F X$ is a homotopy equivalence.

In [10], the author introduced the notion of absolute proper retract (APR). The next result shows how our current work relates to this topic. We use the fact [10, Theorem 4.2] that $X \in \mathrm{APR}$ if and only if $X$ is noncompact, $F X \in \mathrm{AR}$, and $E X$ is an unstable subset of $F X$.

(4.5) Theorem. Suppose $X \in A N R$. Then $X \in A P R$ if and only if $X$ is noncompact, contractible, and docile at $\infty$.

Proof. If $X \in \mathrm{APR}$, then $X$ is a noncompact AR [10]. Hence $X$ is noncompact and contractible. Furthermore, $F X \in A R$ and $E X$ is an unstable subset of $F X$ so, by Theorem $4.2, X$ is docile at $\infty$. Conversely, if $X$ is noncompact, contractible, and docile at $\infty$, then $F X \in \mathrm{ANR}$ and $E X$ is an unstable subset of $F X$. Furthermore, by Theorem 4.4, the inclusion of $X$ into $F X$ is a homotopy equivalence, so $F X$ is contractible, hence an AR. By Theorem 2.1, $X \in A P R$.

\section{BIBLIOGRAPHY}

1. B. J. Ball, Proper shape retracts, Fund. Math. 89 (1975), 177-189.

2. B. J. Ball and R. B. Sher, A theory of proper shape for locally compact metric spaces, Fund. Math. 86 (1974), 163-192. MR 48 \#12458.

3. K. Borsuk, Theory of retracts, Monografie Mat., Tom 44, PWN, Warsaw, 1967. MR 35 \#7306.

4. S. Eilenberg and R. L. Wilder, Uniform local connectedness and contractibility, Amer. J. Math. 64 (1942), 613-622. MR 4, 87. 
5. H. Freudenthal, Neuaufbau der Endentheorie, Ann. of Math. (2) 43 (1942), 261 279. MR 3, 315.

6. S.-T. Hu, Theory of retracts, Wayne State Univ. Press, Detroit, Mich., 1965.

MR 31 \#6202.

7. G. Kozlowski, Images of ANR's, Trans. Amer. Math. Soc. (to appear).

8. K. Morita, On images of an open interval under closed continuous mappings, Proc. Japan Acad. 32 (1956), 15-19. MR 18, 140.

9. R. B. Sher, Property SUV ${ }^{\infty}$ and proper shape theory, Trans. Amer. Math. Soc. 190 (1974), 345-356. MR 49 \#6140.

10. - A theory of absolute proper retracts, Fund Math. 88 (1975), 241-247.

DEPARTMENT OF MATHEMATICS, UNIVERSITY OF NORTH CAROLINA AT GREENSBORO, GREENSBORO, NORTH CAROLINA 27412 